\title{
SISTEMA DE INFORMACIÓN PARA \\ LA INVESTIGACIÓN UNADISTA
}

\author{
Juan Carlos Hernández Barrero ${ }^{35}$
}

\section{RESUMEN}

El presente artículo describe el proceso de evolución de un Sistema de Información implementado en el proyecto de investigación "Visibilidad y Desarrollo Científico, ¿Quién es quien en investigación en la UNAD? Presenta una descripción de sus componentes, explica el impacto en el proceso investigativo, su estructura operacional y enfatiza en el uso de los desarrollos tecnológicos en materia de administración y gestión de la información.

Palabras clave: sistema de información, desarrollo tecnológico, sociedad del conocimiento, información, innovación, investigación.

\begin{abstract}
This article presents the evolution of an information system created as part of the research project "Scientific Visibility and Development- The Who's Who in Research at UNAD". It describes its components, explains its impact on the research process, its operational structure, and emphasizes the application of Information Technology innovation to information management.
\end{abstract}

Key words: information system, technological development, Knowledge society, innovation, research.

\section{INTRODUCCIÓN}

La dinámica de la sociedad de la información precisa activar estrategias que apoyen los procesos de administración de la masa de datos que día a día emergen; una actividad muy exitosa es la implementación de Sistemas de Información (sI), en la cual se apoyan los procesos investigativos, en la actualidad, para los ejercicios de consolidación de la información, optimizando así presupuesto y tiempo.

Este artículo describe el desarrollo y validación de un Sistema de Información para la gestión de la visibilidad de los actores de la investigación institucional en la UNAD. Dicho sistema se desarrolló en tres etapas que relatan la evolución del proceso de construcción; la primera

35 Licenciado en Diseño Tecnológico, candidato a Magister en Tecnologías de la Información aplicadas a la educación, docente investigador de la Escuela de Ciencias de la Educación en la Universidad Nacional Abierta y a Distancia, miembro del grupo de investigación Gestión Vital registrado y clasificado en categoría C en Colciencias. 
fundamentada en cuestionarios impresos, la segunda usando una base de datos en Access de Microsoft Office, y la tercera con una base de datos interactiva y en línea programada en PHP.

La información que se registra en el si es sobre datos personal, formación académica, participación, producción investigativa; además, se plantearon allí preguntas referentes al interés por la investigación, las competencias investigativas que se han desarrollado en los procesos en los que ha participado y una postura personal frente a si la UNAD es un escenario propicio para desarrollar investigación. Para la validación de dicho Sistema de Información se hizo una prueba con el grupo de investigadores del proyecto, esta permitió realizar ajustes a las preguntas y dar paso a su aplicación piloto en el CEAD José Acevedo y Gómez de la zona de Bogotá-Cundinamarca.

Para la primera base de datos se hizo una revisión de las respuestas del cuestionario impreso, se ajustaron algunas preguntas y se generaron categorías a partir de las respuestas de los participantes. Con los ajustes se implementó el Sistema de Información en medio digital en Microsoft Access y se hizo la validación con participantes de diferentes centros. El cuestionario en línea atiende a los requerimientos de cobertura y acceso remoto, se optimiza en el dimensionamiento de los campos de los registros ya que presentaba algunos problemas de capacidad de caracteres en el almacenamiento de los datos.

\section{MARCO TEÓRICO}

A continuación se presentan algunos referentes teóricos que sustentan el trabajo que se ha venido desarrollando en el diseño e implementación del Sistema de Información (SI) para la gestión de la investigación en la UNAD.

\section{Sistema}

Como primer elemento se considera el concepto de sistema y se entiende como un conjunto de componentes que unidos entre sí cumplen un objetivo específico. Un sistema, en general, tiene una entrada, un proceso y una salida. La entrada se refiere a los insumos que se necesitan para trabajar, el proceso se encarga de transformar dichos insumos en un producto nuevo a la salida. Este producto debe cumplir con los objetivos del sistema. Para asegurar este cumplimiento se realiza una retroalimentación constante que pretende ajustar el proceso en sí para que cumpla con el objetivo. Todo lo que rodea al sistema se denomina medio ambiente. Para que un sistema subsista este debe ser capaz de interactuar constantemente con el medio ambiente.

Piatini, Calvo, Cervera y Fernández, (2004) retoman la definición de sistema de la Real Academia de la Lengua Española que nos dice que sistema es un conjunto de cosas que, relacionadas entre sí ordenadamente, contribuyen a un determinado objetivo (RAE, 2001); y a partir de esta definición identifican como los principales elementos presentes en cualquier sistema los siguientes:

- Los componentes del sistema

- Las relaciones entre ellos que determinan la estructura del sistema

- El objetivo del sistema 
Adicionalmente a los elementos que se encuentran presentes en un sistema es importante definir el concepto de subsistema. Cada parte del sistema se denomina subsistema como un sistema inmerso capaz de colaborar en la consecución del objetivo del sistema. Por ejemplo, en el sistema universidad existen como subsistemas las diferentes facultades, unidades administrativas, sistema de investigación, biblioteca, etc., donde todos colaboran para que la universidad cumpla su objetivo.

Gráfica 1. Dinámica del sistema

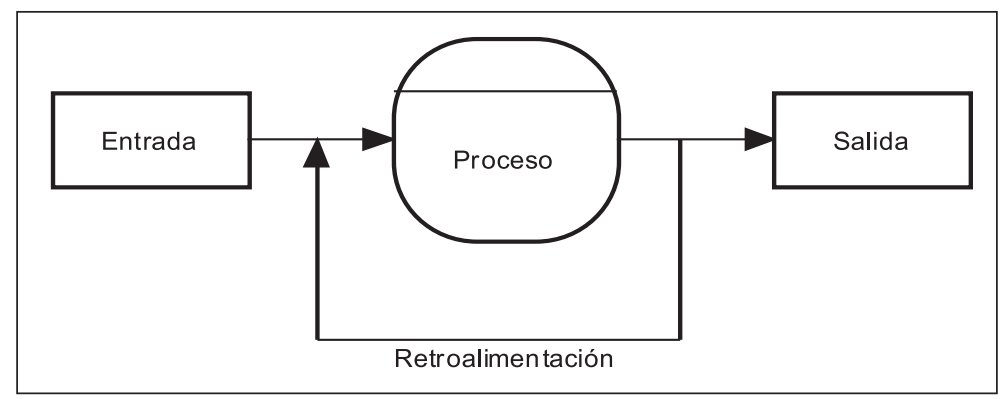

\section{Sistema de Información}

Posterior a la contextualización del concepto de Sistema y los elementos que interactúan en este (Subsistemas), se aborda el Sistema de Información y se dice que es aquel sistema diseñado para recoger, clasificar, analizar, procesar y almacenar información que posibilite la toma de decisiones dentro de una organización.

Piatini, et.al., (2004) definen un SI como, un conjunto formal de procesos que, operando sobre una colección de datos estructurada según las necesidades de la empresa, recopilan, elaboran y distribuyen la información (o parte de ella) necesaria para las operaciones de dicha empresa y para las actividades de dirección y control.

$\mathrm{Al}$ atender las múltiples definiciones de sistema se hizo evidente que para el buen manejo de la información obtenida en el desarrollo del proyecto era necesario un Sistema de Información, y concentrados en la definición de sistema de Piatini, et.al., (2004) que se acaba de exponer, el grupo Gestión Vital tomó la decisión de desarrollar un Sistema de Información que estuviera acorde y fuera correspondiente con las necesidades de tratamiento y administración de los datos recolectados en el desarrollo de la investigación.

\section{Innovación}

El concepto de innovación, en el presente artículo, se entiende como la incorporación de nuevo un elemento que entra a dinamizar el proceso investigativo, incluye acciones de registro para habilitar la visibilidad de los actores y sirve como medio de comunicación de los sentires personales en términos de investigación. 
En esta lógica, el si objeto de estudio del presente artículo, se considera una innovación del producto y del proceso, como lo plantea Michael Porter. El primero, por incorporar un elemento tecnológico mejorado que genera cambios satisfactorios al sistema, y el segundo, por originar cambios significativos en el sistema de dirección, organización y calidad.

Se ha entendido esta innovación como un elemento gestor de la sociedad de la información que fortalece los procesos de administración. En este estudio se consibe el si como una incorporación del sistema que influye en la toma de decisiones, en el reporte de informes, en la consolidación de bases de datos y en la evolución de un sistema de investigación que se nutre de los desarrollos tecnológicos en su proceso de evolución.

\section{Sociedad del conocimiento}

Nonaka y Konno (1998) anclan su modelo de comunidad creadora de conocimiento en el concepto de "ba" o espacio para las relaciones sociales que emergen; quienes participan trascienden los límites de su propia perspectiva individual y desarrollan conocimiento en la medida en que comparten con sus pares. Como consecuencia, se generan resultados del colectivo que son superiores a la simple suma de conocimientos individuales porque el conocimiento de la comunidad es resultado de las relaciones emergentes.

Según estos autores, la comunidad creadora de conocimiento funciona a manera de un sistema adaptativo complejo que cambia su configuración como una entidad fractal sobre la base de las contribuciones específicas y es capaz tanto de atraer como de seleccionar: no es cerrada pero tampoco totalmente abierta. Al ser una comunidad puede ser física, pero también virtual.

Esta dinámica genera grandes producciones de información que exigen nuevas estrategias de sistematización, administración, procesos de análisis y generación de reportes. La sociedad del conocimiento, en su dinámica de crecimiento, hace uso de sistemas de información para gestionar sus procesos. Este es el tema principal que se relaciona en este estudio.

\section{SISTEMA DE INFORMACIÓN PARA LA INVESTIGACIÓN DE LA UNAD}

\section{Aplicación}

Al tener en cuenta el proyecto "Visibilidad y Desarrollo Científico, ¿Quién es quien en investigación en la UNAD?, que busca identificar a los actores que desarrollan investigación en esta Universidad, se implementa un Sistema de Información que juega un papel importante en la administración de la información en el campo de la investigación en dicho contexto.

Las exigencias sociales, la gestión del conocimiento es uno de ellas, hacen que la administración de la investigación en la universidad tenga en cuenta diferentes formas de organizar sus desarrollos. Grant (1991) y Schoemaker (1992), proponen el uso adecuado de la información y, en especial, del conocimiento como la principal fuente de diferenciación en un mercado 
de la gestión del conocimiento en la nueva economía cada vez más competitivo y global. Para estos autores, dentro de los recursos que cada organización posee, cabría distinguir entre los recursos tangibles — capital, mano de obra y tierra- y los recursos intangibles o capacidades - mezcla de habilidades y conocimientos que la organización posee. De hecho, algunos investigadores sugieren que la principal ventaja organizativa proviene de la creación, obtención, almacenamiento y difusión el conocimiento, (Nahapiet y Ghoshal, 1998).

En esta lógica el proyecto diseña, desarrolla y aplica un Sistema de Información que atiende las necesidades específicas de la universidad en investigación. Dicho Sistema dinamiza la búsqueda de los datos que el grupo investigador precisa para hacer los análisis requeridos por el proceso investigativo y para entender los fenómenos presentados en la investigación de la UNAD.

Para ir dando forma a la estructura del sistema de investigación de la UNAD se tuvieron en cuenta los elementos que componen un Sistema de Información, pues cada SI se desarrolla de acuerdo con las necesidades del sistema para el que ha sido creado y, por tanto, sus elementos pueden variar en:

- La información: en este ámbito podemos decir que la información es la abstracción que los seres humanos pueden hacer de una colección de datos. Además, es en sí misma un elemento principal y fundamental para el sistema, pues es el que le da vida y utilidad. Las personas involucradas en el sistema deben aprender el manejo de la información disponible y hacerlo lo más eficiente y seguro posible.

- Procedimientos: son aquellas guías y planteamientos que las personas involucradas en un SI deben tener en cuenta para el desarrollo de sus actividades y la buena gestión de los datos. Esto con el fin de garantizar la seguridad, la integridad y la buena administración de la información.

- Usuarios: son todas aquellas personas o entes que introducen, procesan o extraen para su uso la infamación existente en el si.

- Equipo de soporte: con el fin de llevar acabo el trabajo sobre los datos y obtener la información necesaria para el desarrollo del negocio o actividad en curso, se deben emplear elementos de varios niveles de tecnología; archivos físicos, documentación, máquinas, ordenadores y un componente importante por su eficacia en la administración de la información: el Software.

Se toma como referente algunas definiciones de los sistemas de información que orientaron la clasificación del si implementado en el proyecto en cuestión.

- MIS, de Management Information System, Sistema de Información para la gestión. Este tipo de si está desarrollado para la ayuda en la toma de decisiones a nivel de dirección.

- DSS, Decision Support System, Sistema de apoyo a decisiones. Este tipo de SI está diseñado para dar soporte en la toma de decisiones sobre la información poco estructurada, y sin procesos bien definidos. 
- EIS, Executive Information System, Sistema de Información para ejecutivos. Sistema dedicado a mostrar la información relevante del negocio, aquí se encuentran los sistemas que mediante unas pocas gráficas o indicadores pueden mostrar al usuario los aspectos importantes y de cuidado que permiten ver el estado del negocio.

- TPS, Transaction Prossesing Systems, Sistema de procesamiento de transacciones. Este tipo de sistema es el encargado de automatizar las transacciones operativas que se hacen de manera rutinaria sobre datos perfectamente estructurados.

En esos términos, el Sistema de Información desarrollado por el equipo de trabajo es un MIS (Management Information System). La estructura que lo soporta es la siguiente:

Gráfico 2. Estructura del Sistema de Información

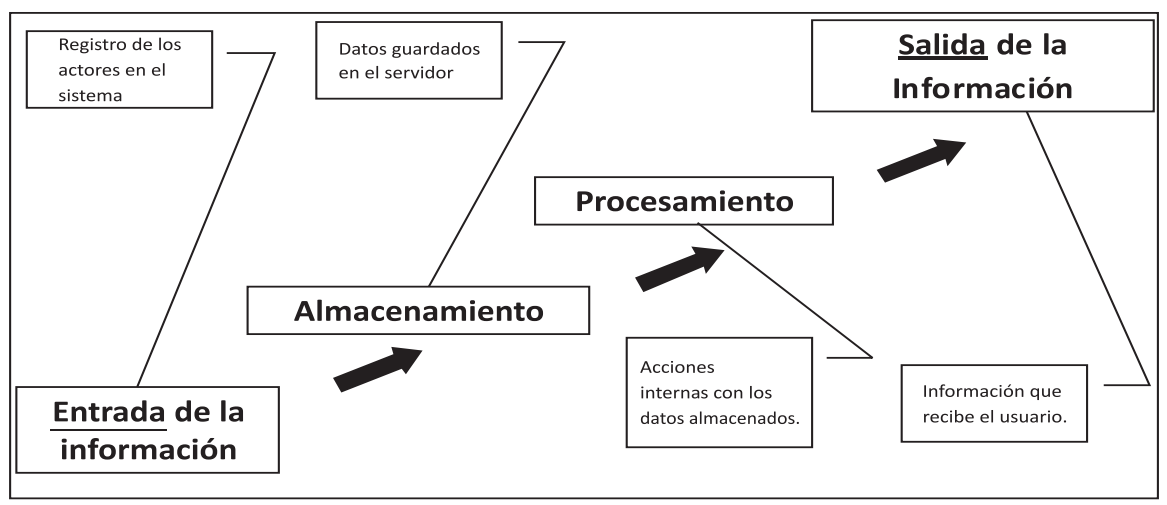

El si desarrollado en la investigación muestra cuatro componentes; la entrada de la información, el almacenamiento, el procesamiento y la salida de la información.

\section{- Entrada de la Información}

En primera instancia, el Sistema de Información realiza un proceso de entrada que consiste en el ingreso de los datos. En el caso particular del si de la presente investigación se registran campos relacionados con la información personal de los investigadores estudiantes y de investigadores tutores, estos primeros presentan un registro en cuanto a: Información personal (identificación), vinculación a la UNAD, formación académica, proyectos de extensión a la comunidad, proyectos de investigación y publicaciones. Y de tutores: información personal (identificación), vinculación a la UNAD, formación académica (pregrado, postgrado), producción investigativa: (semilleros, proyectos de grado, proyectos de extensión a la comunidad, proyectos de investigación, publicaciones). Esta información está relacionada directamente con los objetivos mismos del proyecto y los intereses particulares de la Universidad.

\section{- Almacenamiento de información}

Los datos registrados por los investigadores en el si son guardados en una base de datos en un servidor de la universidad. Esta información se constituye en la base de datos de los investigadores de la UNAD. 


\section{- Procesamiento de Información}

Este componente permite hacer los cruces de información para obtener los resultados requeridos en los diferentes análisis desarrollados.

\section{- Salida de Información}

El proceso de salida de la información en el si, presenta un historial del registro hecho por el usuario, estos datos se especifican a continuación.

\section{Toma de datos}

Para realizar la toma de datos como se mencionó anteriormente se tuvieron en cuenta los actores de la UNAD que estuvieran vinculados con procesos investigativos. A continuación se hace una descripción de los datos de los investigadores docentes que componen el Sistema de Información.

\section{Identificación}

Nombres y apellidos, número de identificación, edad, número telefónico, ciudad de residencia, correo electrónico de contacto.

\section{Vinculación}

Año de vinculación, el tipo de vinculación, el tiempo de dedicación, la escuela, el programa académico, el CEAD, el número de cursos, el número de grupos, número de estudiantes, especificación de los cursos que dirige en investigación, las competencias que pretende desarrollen sus estudiantes en investigación, una postura personal frente a la UNAD como un escenario propicio para desarrollar investigación y evaluar si tiene alguna meta personal en investigación en la UNAD.

\section{Estudios}

Pregrado: título profesional, la universidad que otorgó el título, año de graduación, si presentó trabajo de grado, las competencias en investigación que desarrolló en sus estudios de pregrado, si ha desarrollado trabajos o publicaciones derivados de su proyecto de pregrado.

\section{Postgrado}

Especialización: nombre de la especialización, la universidad que otorgó el título, año de graduación, título de la monografía de grado, las competencias en investigación que desarrolló en sus estudios de especialización, si ha desarrollado proyectos derivados de su trabajo de grado en la especialización y si su trabajo de grado dio origen a alguna publicación.

Maestría: nombre de la maestría, la universidad que otorgó el título, año de graduación, título de la tesis de grado, las competencias en investigación que desarrolló en sus estudios de maestría, si ha desarrollado otros proyectos derivados de su tesis de magíster y si su trabajo de grado dio origen a otras publicaciones. 
Doctorado: nombre del doctorado, la universidad que otorgó el título, año de graduación, título de la disertación doctoral, competencias que considera desarrolló en sus estudios doctorales, si ha generado otros proyectos derivados de su tesis doctoral y si la tesis doctoral ha derivado otras publicaciones.

\section{Producción investigativa}

Semilleros: si orienta algún semillero, el nombre del semillero, el número de miembros, la fecha de iniciación, las líneas de investigación o temática, si ha realizado alguna publicación derivada de la orientación del semillero y cuanto tiempo dedica a la orientación del semillero.

Proyectos de grado: si dirige proyectos de grado, si estos proyectos están inscritos a las líneas de investigación de las escuelas, cuántos proyectos dirige, que relacione los proyectos de excelente calidad y que pueden dar origen a publicaciones y si ha realizado alguna publicación a partir de la dirección de proyectos de grado.

Proyectos de extensión a la comunidad: si está vinculado a algún proyecto institucional o de extensión a la comunidad, la modalidad del proyecto, si el proyecto tiene aprobación de financiación, si está terminado o en ejecución y si tiene alguna publicación derivada de estos proyectos.

Proyectos de investigación: si actualmente tiene inscrito algún proyecto de investigación, si ha sido presentado a alguna convocatoria, si tiene financiación, si está terminado o en ejecución y si tiene alguna publicación derivada.

Publicaciones: si tiene publicados libros de texto, libros de investigación, artículos de investigación y si tiene alguna patente registrada. Finalmente aparece una pregunta que relaciona el interés por la participación en el taller de redacción de artículos científicos.

El diseño de los formularios busca obtener los datos que se consideran relevantes para obtener información valiosa en coherencia con la dinámica de la visibilidad en el proceso investigativo.

\section{Cómo sacarle provecho al SI}

El Sistema de Información, para la investigación en la UNAD, es una herramienta que evidencia la ayuda que ofrecen las Tic en los procesos académicos y específicamente en la investigación en la Universidad. La implementación de este software ha revolucionado los procesos de recolección de la información, atiende claramente a la infraestructura de la institución y efectivamente a las necesidades particulares del proyecto. Una de las grandes exigencias presentadas por el Sistema de Información para sacarle el mejor provecho es la actualización constante, ya que es una herramienta que almacena la información y permite inferir directamente sobre la dinámica de la investigación en la UNAD. 
La idea es generar estrategias que permitan mantener el Sistema de Información con los datos actualizados, para que los actores investigativos de la institución no desconozcan su existencia y su alcance, y para hacer el mejor uso posible de esta poderosa herramienta.

\section{Ejemplificación}

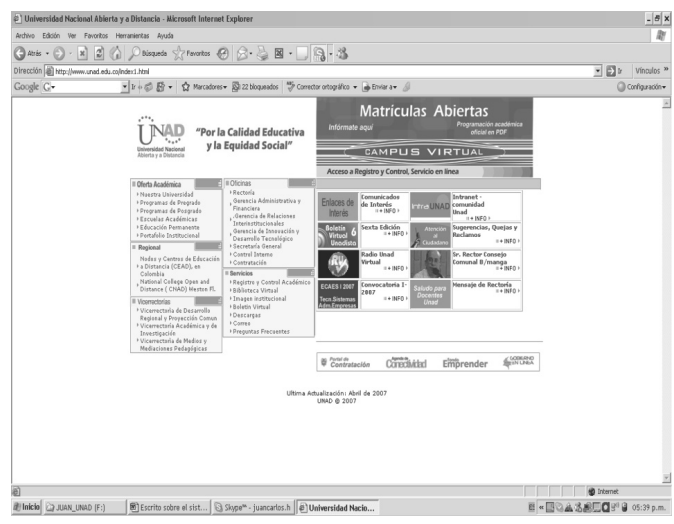

Figura 1. El Sistema de Información I

El Sistema de Información permite acceder desde la Intranet de la UNAD, www.unad.edu.co Sistema de Investigación, link de Sistema de Información.

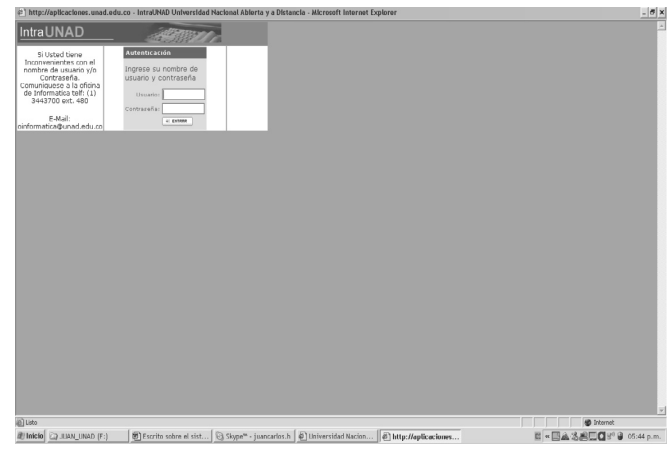

Figura 2. El Sistema de Información II

El Sistema de Información está orientado a aquellas personas que cumplan con el perfil de investigador, los interesados solicitan el nombre de usuario y contraseña al E-Mail: siunad@ unad.edu.co 


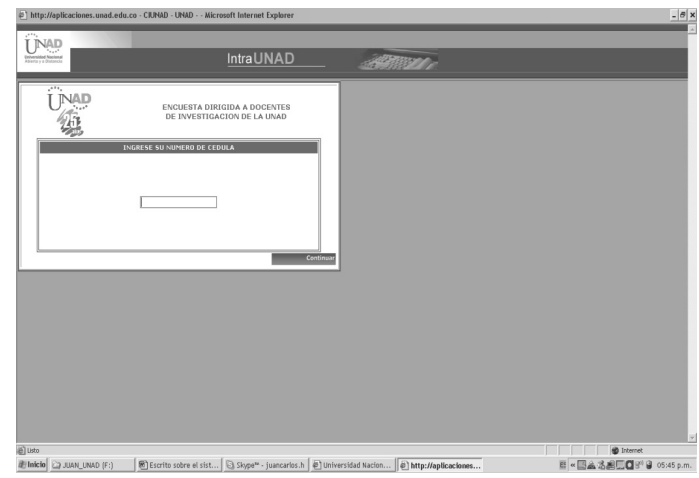

Figura 3. El Sistema de Información III

El sistema solicita el número de identificación, con este número se puede referenciar para cualquier búsqueda específica.

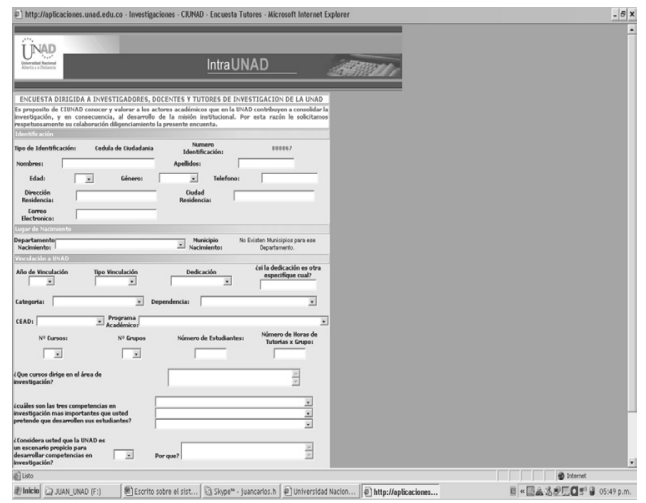

Figura 4. El Sistema de Información IV

Por último se procede a ingresar la información en el software que irá orientando el progreso de la captura de los datos de los investigadores.

\section{CONCLUSIONES}

En los tiempos actuales, con los fenómenos del crecimiento incontrolado de la información, el uso de desarrollos tecnológicos, como es el caso de un Sistema de Información, posibilita obtener mejores resultados en materia de administración y gestión de la información.

La implementación de un Sistema de Información en el proyecto de "Visibilidad y Desarrollo Científico, ¿Quién es quién en investigación en la UNAD?, ha sido una estrategia efectiva en la consolidación de la base de datos de investigadores de la UNAD, y ha optimizado la 
administración de la información brindando mejores condiciones a los investigadores para el proceso de análisis de los datos estadísticos.

Es de vital importancia mantener en observación el Sistema de Información para la investigación de la UNAD, con miras a realizar desarrollos tecnológicos que apunten a la optimización de los procesos investigativos de la universidad.

Una persona que participe de las estrategias de los sistemas de información tiene más posibilidades de hacerse visible ante una comunidad que una que no participe de la estrategia.

Los desarrollos tecnológicos posibilitan a las personas el acceso a diferentes dinámicas sin importar distancias o ubicaciones geográficas.

\section{REFERENCIAS BIBLIOGRÁFICAS}

ANDREU, R., Ricart, J. \& VALOR, J. (1996). Estrategia y sistemas de información. Bogotá, Colombia: McGraw Hill.

BERTALANFFY, Ludwig Von. General System Theory: Foundations, Development, Applications. Revised Editles 1976.

CANO, Jeimy. Critical Reflections on Information Systems: A Systemic Approach. Hershey, PA, USA: Idea Group Inc., 2002. P. 200. http://site.ebrary.com/lib/unad/ Doc?id=10019201\&ppg=213.

CERDA, Hugo. Cómo Elaborar Proyectos. Santafé de Bogotá: Magisterio, 1997. 110 p.

DELGADO, Magola. Revista Colombia Ciencia y Tecnología. Divulgación Científica: La Distribución del Saber. Bogotá: Colciencias Vol. 11 No. 4 Octubre de 1993.

HURTUBISE, R. (1984). Managing information systems: concepts and tools. West artford, CT, Kumarian Press.

NAHUIS, Richard (2004). Learning for Innovation and the Skill Premium Jounal of Economics, Vol. 83 (2004), No. 2, pp. 151-179.

MALDONADO, Luis. Metacognición y razonamiento espacial en juegos de computador. 1999. $226 \mathrm{p}$.

MARTÍNEZ, Eduardo. LA Popularización de la Ciencia y la Tecnología. México: UNESCO 1997. $106 \mathrm{p}$.

PIATTINI V, José A. CALVO, Juaquin CERVERA B, Luis Fernandez S. Análisis y diseños de aplicaciones informáticas de gestión, una perspectiva de la ingeniería de software. Editorial Alfa Omega. 
SENN, James A. Diseño de sistemas de información. Mc. Graw Hill (Mexico) ISBN: 968422-991-7, 1990.

TAICHI, Sakaiya. Historia del Futuro: La Sociedad del Conocimiento. Santiago de Chile:

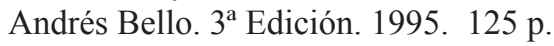

TREJO DELARBRE, Raúl. 2.000 Revista iberoamericana de ciencia, tecnología, sociedad e innovación. OEI, Vivir en la Sociedad de la Información, Orden global y dimensiones locales en el universo digital.

WHITTEN, JEFFREY L. Systems Analysis and Design for the System Enterprise. McGraw Hill Higher Education 2006, ISBN: 9780071107662. 